\title{
Auf den Klang kommt's an: Die Vertonung eigener Geschichten zur intensiven Auseinandersetzung mit Prosodie im DaF- Unterricht
}

Denise Gensel

Universidad de Sevilla

dgensel@us.es

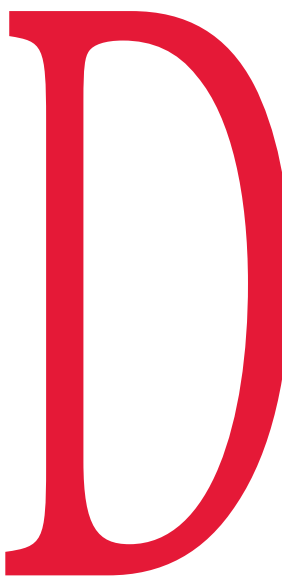

iese (die Umlaute $\ddot{A}$, Ö und Ü) waren und sind meine größten Feinde auf Erden, was die Aussprache betrifft. Bis heute machen mich diese Laute nervös. Schon mehrere Silben vor einem solchen Umlaut wird meine Zunge ganz unruhig, werden meine Lippen trocken. Diese phonetischen Tretminen sind für mich so, als kaute ich auf Nägeln. Als hätte man sie in die Worte geschlagen, die ich bilden möchte, und als verhinderten sie wie Widerhaken, dass diese meinen Mund verlassen (Khider 2019: 19).

Dass die Artikulation bestimmter Laute des Deutschen, die der Schriftsteller Abbas Khider in seinem Buch Deutsch für alle als „phonetische Tretminen“ bezeichnet, mit Schwierigkeiten verbunden sein kann, dürfte den meisten DaF-Lerner*innen aus ihrer eigenen Sprachlernbiografie bekannt sein. Vor allem die im muttersprachlichen Lautinventar nicht vorhandenen Laute und Lautkombinationen stellen häufig ein Hindernis beim flüssigen Sprechen dar, werden manchmal gar als unangenehm empfunden. Artikulatorische Normabweichungen beschränken sich jedoch nicht auf Einzellaute, sondern betreffen auch den suprasegmentalen Bereich, dessen Thematisierung in DaF-Lehrwerken einen vergleichsweise geringen Raum einnimmt. Dabei zeigen Forschungsergebnisse, welch hohen Stellenwert prosodische Merkmale für die Verständlichkeit lernersprachlicher Äußerungen, die Hörerorientierung in monologischen Reden sowie die Sprechwirkung aufseiten der Hörerinnen einnehmen. Ein Fokussieren im DaF-Unterricht ist dabei schon auf perzeptiver Ebene anzusetzen. Denn die durch die Muttersprache modellierten Hörmuster beeinträchtigen die Wahrnehmung unbekannter Segmente und Suprasegmentalia, die in der Ausgangssprache keine Systemrelevanz haben und führen dazu, dass diese schlicht „überhört“ und deshalb selten korrekt produziert werden können. Dies macht Hörtrainings in Form von diskriminierenden und identifizierenden Hörübungen im Fremdsprachenunterricht unabdingbar.

Wird sich im DaF-Unterricht dem Thema Prosodie gewidmet, so meist durch ein wenig breit gefächertes Übungsformat (mit Pfeilen die Melodieführung am Äußerungsende oder den Wortakzent durch Unterstreichen einzelner Silben markieren). Der vorliegende Beitrag unterbreitet den Vorschlag, DaF-Lernerinnen durch die Vertonung von Geschichten für prosodische Merkmale zu sensibilisieren, zu emotionalem Sprechen anzuregen und sich so einem Bewusstwerden der eigenen Sprechwirkung anzunähern. 


\section{Prosodische Merkmale und ihre Relevanz}

Unter dem Begriff Prosodie sind alle Einzellaute überlagernden Eigenschaften gesprochener Sprache gefasst. Dazu zählen Tonhöhenverlauf, Lautstärke, Sprechgeschwindigkeit, Dauer, Pausen, Sprechspannung sowie Stimmklang. Ihre Eigenschaft, stets an Einzellaute geknüpft zu sein, erschwert eine isolierte Betrachtung dieser auch als Suprasegmentalia bezeichneten Phänomene im Fremdsprachenunterricht. Zudem treten prosodische Merkmale meist in Kombination auf und erzeugen so die Rhythmisierung und Akzentuierung gesprochener Sprache.

Die phonetische Forschungslandschaft hält mehrere Studien bereit, die die Bedeutung jener klanglichen Eigenschaften des Deutschen empirisch belegen. So wurde bestätigt, dass Normabweichungen auf prosodischer Ebene das Verständnis von gesprochenen Äußerungen erheblich beeinträchtigen und dies sogar teilweise stärker als Fehler im lautlichen Bereich. Hirschfeld wies die enorme Relevanz von Wort- und Satzakzent in einer Perzeptionsstudie nach. Der Satz Es, regnet. wurde deutschen Hörer*innen mit einem falschen Akzentmuster vorgespielt (Es regnet.). Die hörerseitige Rezeption ist durch das Streben, dem Wahrgenommenen Sinnhaftigkeit zu entnehmen bzw. zu verleihen, gekennzeichnet und das Gehörte kann dafür Korrekturprozessen unterzogen werden. Nur 48,8\% der Proband*innen haben in Hirschfelds Studie den Satz Es ,regnet. erkannt, waren also in der Lage, als Korrekturleistung den Wortakzent zu variieren. Gut die Hälfte hingegen gab an, Sätze wie Ist sehrnett. Geh ins Bett! Willst du mit? gehörtzu haben, also vom Ausgangssatz stark abweichendes Sprachmaterial, dass jedoch die gleichen Akzentuierungsmuster aufweist (Vgl. Hirschfeld 1995). Über die Verständlichkeit hinaus kommt sprechstimmlichen Parametern eine redestrukturierende und hörerorientierende Funktion zu (Vgl. GrzeszczakowskaPawlikowska 2016). Zudem üben sie Einfluss auf die hörerseitige Behaltensleistung aus. Demnach führt eine intensiv gestaltete Sprechweise dazu, dass sich Hörer*innen an mehr Informationen eines gesprochenen Textes erinnern können als bei einer monotonen Vortragsweise (Vgl. Neuber 2006). Auch ob sich eine Person wohlfühlt, sie aufgeregt, verschlafen, euphorisch oder zutiefst niedergeschlagen ist, offenbart nicht zuletzt ihre Stimme.

Aus Hirschfeld/Reinke lässt sich folgende zusammenfassende Übersicht entnehmen:

\begin{tabular}{|c|c|}
\hline syntaktische Funktion & $\begin{array}{l}\text { Kennzeichnung der zugrunde liegenden syntaktischen } \\
\text { Strukturen }\end{array}$ \\
\hline strukturierende Funktion & $\begin{array}{l}\text { Gliederung längerer Äußerungen in inhaltlich } \\
\text { zusammengehörige Teiläußerungen }\end{array}$ \\
\hline kommunikative Funktion & $\begin{array}{l}\text { Kennzeichnung/Hervorhebung wichtiger Informationen } \\
\text { gegenüber weniger wichtigen; Steuerung von } \\
\text { Gesprächsabläufen (z.B. den Sprecherwechsel) }\end{array}$ \\
\hline markierende Funktion & $\begin{array}{l}\text { Kennzeichnung regional, soziophonetisch und } \\
\text { phonostilistisch geprägter Sprechweisen }\end{array}$ \\
\hline expressive oder affektive Funktion & $\begin{array}{l}\text { Kennzeichnung von Emotionen und subjektiven } \\
\text { Befindlichkeiten der sprechenden Person }\end{array}$ \\
\hline
\end{tabular}

Hirschfeld/Reinke 2018: 58

Darüber hinaus hat eine Übertragung von Artikulationsgewohnheiten aus der Muttersprache in die Fremdsprache die Entstehung eines fremden Akzents zur Folge. Die Interferenzen liegen dabei sowohl im lautlichen Bereich als auch auf prosodischer Ebene. In Sprechwirkungsstudien konnte ein Zusammenhang zwischen dem Ausprägungsgrad des fremden Akzents und einer Persönlichkeitsbewertung der Sprecher*innen festgestellt werden (Vgl. Reinke 2011, Reinke 2007). Der Umstand, dass sich dies gar negativ auf die Persönlichkeitsbeurteilung durch Hörerinnen mit Deutsch als Muttersprache auswirken kann (Vgl. Cohrs 2007), unterstreicht die Dringlichkeit, dem Thema Sprechwirkung im DaFUnterricht Raum zu geben. Schließlich gilt es als ein wesentliches kommunikatives Anliegen von Individuen und somit auch DaF-Lernerinnen, dass das von ihnen vermittelte Fremd- mit ihrem Selbstbild weitestgehend kongruent ist.

Daher müssen sowohl die emotionalen Intentionen als auch die erreichten Wirkungen bewusstgemacht, die Lernenden für die entsprechenden phonetischen Ausdrucksformen sensibilisiert und deren Anwendung trainiert werden. (Hirschfeld/Reinke 2018: 143) 


\section{Interferenzphänomene bei DaF-Lerner ${ }^{\star}$ innen mit Spanisch als Muttersprache}

Doch wo genau liegen die Interferenz-Gefahr bergenden Unterschiede im klanglichen Bereich zwischen den Sprachen Deutsch und Spanisch? Die nun folgende Skizzierung der prosodischen Differenzen erhebt keinerlei Anspruch auf Vollständigkeit und Allgemeingültigkeit. Nicht nur das jede ${ }^{\star} r$ Lernerin eine individuelle Sprachlernbiografie mitbringt, auch lässt sich eine Vielzahl an (regionalen) Varietäten des Spanischen finden, deren Artikulationsgewohnheiten voneinander abweichen können.

Das Spanische weist klare Regeln der Wortakzentuierung auf. Wörter, die auf Vokal oder den Konsonanten $n$ oder $s$ enden, werden auf der vorletzten Silbe betont (palabras llanas). Bei allen anderen erfolgt die Wortakzentuierung auf der letzten Silbe (palabras agudas). Abweichungen von dieser Verteilung wie beispielsweise die häufige Betonung der drittletzten Silbe (palabras esdrújulas) werden durch einen Akut gekennzeichnet. Im Deutschen hingegen ist die Wortakzentuierung weitaus weniger offensichtlich. Es besteht im Gegensatz zum Spanischen eine Tendenz zur Anfangsbetonung. Zudem können auf eine Akzentsilbe mehrere nicht akzentuierte Silben folgen (z.B., hoffentlich). Dieses Phänomen wiederum lässt sich auch als rhythmische Besonderheit des Deutschen beschreiben. Zu fehlerhaften Realisierungen kann es zudem bei der Kombination Wortakzent und kurzer Silbenvokal kommen. Häufig wird dieser dann zu lang realisiert (z.B. ,Sonne). In beiden Sprachen kommt dem Wortakzent eine bedeutungsunterscheidende Funktion (z.B. ,umfahren vs. umfahren; ,August vs. Au'gust) zu. Ein Rückgriff auf Minimalpaare bietet sich demnach beim Üben an.

In Bezug auf den Rhythmus ist der Gegensatz zwischen dem relativ ausgeglichenen Wechsel zwischen akzentuierten und nicht akzentuierten Silben im Spanischen sowie dem für das Deutsche charakteristischen Phänomen, dass auf eine akzentuierte Silbe mehrere nicht akzentuierte Silben folgen können, die einer starken Reduktion unterworfen sind, hervorzuheben. Oftmals fällt dieser Kontrast zu schwach aus. Es ist also eine zu geringe Sprechspannung zu verzeichnen. Valman untersuchte in diesem Zusammenhang die Schwierigkeiten, die unbetonte Silben spanischsprachigen DaFLerner*innen bereits auf der perzeptiven und daraus resultierend auf der produktiven Ebene bereiten (Vgl. Valman 2016).

Auch bei der melodischen Gestaltung sind unterschiedliche Kodierungsgewohnheiten zu verzeichnen. So variiert der Tonhöhenverlauf im Spanischen weniger als im Deutschen (Vgl. Hirschfeld 2003). Zudem ist die Lösungstiefe, ein für das Deutsche typischer, starker Tonhöhenfall am Äußerungsende, der Abgeschlossenheit und Nachdrücklichkeit markiert, im Spanischen nicht systemrelevant (Vgl. Alvarez-Finkbeiner 2013: 7). Dieses Sinken der Melodie in den untersten Sprechstimmbereich ist im Deutschen allerdings beim Vorlesen von rhetorischer Relevanz und ist bei der akustischen Modellierung von Aufforderungssätzen zu finden.

Die Bewertung des Schweregrades eines Fehlers hängt mit dessen mehr oder weniger störenden Wirkung im konkreten Kommunikationsprozess zusammen. [...] Beispielweise ist es möglich, dass eine eigentlich geringfügige Abweichung (z.B. das Nichterreichen der Lösungstiefe bei gleichzeitiger Intention, sich in einem Bewerbungsgespräch als besonders kompetent darstellen zu wollen) eine sehr negative Auswirkung auf das Kommunikationsergebnis hat und sogar auf die Einschätzung der/ des Sprechenden auswirkt (Hirschfeld/Reinke 2018: 151).

Ein Rückgriff auf kontrastive Fehleranalysen dient vor allem dazu, mögliche Fehlerquellen bei sprachlich homogenen Lerner*innengruppen vorauszusagen und das phonetische Übungsangebot gezielt zu optimieren.

\section{Empfehlungen für den Unterricht}

Wie eingangs bereits erwähnt spielen Hörgewohnheiten aus der Muttersprache eine wesentliche Rolle, die es beim Phonetiklehren zu berücksichtigen gilt. So bringen DaF-Lerner*innen von ihren Ausgangssprachen geprägte Hörmuster in den Lernprozess mit ein, die die Wahrnehmung fremder Laute oder unbekannter prosodischer Phänomene erschweren. Deshalb sollten Lerner*innen zunächst einmal mit Hörtrainings zum Ausbau ihrer auditiven Wahrnehmungsfähigkeit konfrontiert werden. In diese die muttersprachlichen Hörmuster erweiternde Kategorie fallen Übungen der Diskrimination und des Identifizierens von Lauten, Akzentuierungsmustern oder Melodieverläufen. Darauf aufbauend lassen sich Echoübungen mit den Lerner*innen durchführen, dabei handelt es sich um der Automatisierung dienende Übungsformate, bei denen Imitation und Nachsprechen im Vordergrund stehen. Erst dann ist ein Heranführen 
an Formate, bei denen prosodische Mittel selbständig adäquat eingesetzt werden sollen, sinnvoll.

Bei der Thematisierung von Prosodie im Unterricht ist über das bloße Kriterium der Verständlichkeit hinaus und somit der Polarität richtig - falsch ein Bewusstmachen der eigenen Sprechwirkung anzustreben. Lerner*innen sollten damit vertraut werden, dass die prosodische Gestaltung ihrer Äußerungen stets eine Wirkung bei Hörerinnen erzeugt -sowohl in der Mutter- als auch in der Fremdsprache. So kann als Erweiterung der Verständlichkeit die Frage lauten, ob die prosodische Modellierung der Äußerung adäquat in Anbetracht der kommunikativen Absicht erfolgte. Also beispielsweise ob in einem Bewerbungsgespräch Kompetenz und ein gewisses Maß an Selbstvertrauen durch die Sprechweise übermittelt wird. Auch hier ist bereits auf der Ebene der Wahrnehmung anzusetzen. Aussagen können zunächst einmal auf ihre Wirkung hin eingeschätzt werden (z.B. Grad der Höflichkeit, Nachdrücklichkeit etc.) und dies stets unter der Fragestellung, welche prosodischen Mittel wie eingesetzt wurden.

Es ist wünschenswert, dass Lerner*innen im Unterricht genügend Anreiz gegeben wird, sich im emotionalen Sprechen selbst auszuprobieren und nach und nach der vielfältigen Wirkung ihrer Sprechweise(n) bewusst zu werden. Hirschfeld/ Reinke kritisieren diesbezüglich ein Defizit in Lehr- und Lernmaterialien für das Fach Deutsch als Fremdsprache:

Die notwendige Reflexion hinsichtlich einer angestrebten bzw. erzeugten Wirkung (z.B. die Verwendung einer angemessenen höflichen Sprechweise) in der Kommunikation wird nicht angeregt, sodass die notwendige bewusste Angleichung situativ bedingter Sprechweisen nicht erfolgen kann und stattdessen weiter unbewusst auf die gewohnte - aber nicht immer passende Sprechweise aus der Ausgangssprache zurückgegriffen wird (Hirschfeld/Reinke 2018: 185).

\subsection{Die Vertonung eigener Geschichten}

Ein Einsatz von Hörbüchern im DaF-Unterricht wurde bereits bei Travkina befürwortet. So diene die Hörversion literarischer Texte neben dem kulturellen Lernen sowie der Förderung von Hör-, Lese- und Sprechfertigkeit, auch der Sensibilisierung für den Klang der deutschen Sprache und der Erweiterung der durch die Muttersprache modellierten Hörmuster (Vgl. Travkina 2007). Weiterhin schlägt sie vor, Ausschnitte aus Hörbüchern auf prosodische Besonderheiten und deren emotionale Wirkung hin zu analysieren, um im Anschluss die Lerner*innen selbst die literarischen Texte vorlesen zu lassen (Vgl. ebd.).

Im Rahmen eines Seminars zur Förderung der Sprech- und Schreibkompetenz am Germanistischen Institut der Universität Sevilla wurde mit einer Gruppe von Student*innen, deren Deutschkenntnisse auf B1-Niveau lagen, die Vertonung eigener Geschichten angeleitet, um sich auf eine abwechslungsreiche Weise den Themen Prosodie und Sprechwirkungen zu nähern. Dabei erforderte vor allem die Vorbereitungsphase eine intensive Auseinandersetzung mit emotionalem Sprechen, auf perzeptiver, reproduzierender sowie produktiver Ebene. Diese umfasste Hörübungen, in denen die Lerner*innen zunächst einmal vorgespielte Aussagen nach deren Grad an Freundlichkeit/Höflichkeit/ Nachdrücklichkeit zu bewerten hatten. Stets mit der Fragestellung, was bei deren prosodischer Gestaltung jeweils auffällig war. Diese Übung bietet je nach Ausprägung der einzelnen Merkmale (z.B. eine besonders hohe Lautstärke - unhöflich) häufig Potential zur Diskussion. Das ist durchaus erwünscht. Zudem wird ersichtlich, dass es nicht rezeptartig die eine Gestaltungsweise gibt, die zwangsläufig eine freundliche/unfreundliche etc. Wirkung erzielt.

Nach den wahrnehmungsschulenden Aktivitäten übernehmen die Lerner*innen selbst die Rolle der Sprecher*innen. Um ein möglichst breites Spektrum an emotionalen Varianten zu erfahren und zu erproben, eignen sich Aktivitäten aus dem Bereich des szenischen Spiels. Sartor plädierte bereits dafür, die beiden „Stiefkinder" Phonetik und darstellendes Spiel im DaF-Unterricht zu verbinden (Vgl. Sartor 2007). In der Tat hat sich in der Unterrichtspraxis ein spielerischer Umgang mit Prosodie, das Übernehmen anderer Rollen, Imitieren und Nachahmen anderer Sprechweisen und Simulieren von Gemütszuständen und emotionalen Lagen als förderlich erwiesen. Es folgen didaktische Vorschläge diesbezüglich:

- Ein kürzerer Dialog, der der aktuellen Lehrwerkslektion entstammen kann, wird den Lerner`innen in auf A4Format gedruckten Teilen präsentiert. Jede ${ }^{\star}$ r Student ${ }^{\star}$ in erhält eine Äußerung und positioniert sich an der richtigen Stelle, sodass sich die Redeanteile von Gesprächspartner*in A und B versetzt gegenüberstehen. Im Anschluss wird der Dialog mehrmals laut gelesen - jede ${ }^{\star}$ r Sprecher ${ }^{\star}$ in nur seinen ${ }^{\star}$ ihren Satz - und dies auf eine bestimmte emotionale Sprechweise, die entweder das verbleibende Publikum oder die Lehrkraft oder der ${ }^{\star}$ die erste Sprecher ${ }^{\star}$ in selbst vorgibt. Die Lerner*innen haben dabei die Möglichkeit, die Sprechweise des ${ }^{\star}$ der Vorgänger ${ }^{\star}$ in zu übernehmen oder 
in ihrem Schauspiel zu variieren. Die Redebeiträge könnten ebenso gesummt werden. D.h. die Lerner ${ }^{\star}$ nnen lösen sich vom konkreten Sprachmaterial und widmen sich ausschließlich der klanglichen Modellierung. Eine solche Entsprachlichung ist stets angebracht, um zu vermeiden, dass sich Lernerinnen zu sehr auf lautliche Erscheinungen konzentrieren.

- Eine andere Übung sieht vor, kurze Fragen, die auf Kärtchen gezogen werden, auf eine bestimmte emotionale Weise ihrem Gegenüber zu stellen. Dazu bewegen sie sich zu Musik durch den Raum. Sobald die Musik stoppt, präsentiert die Lehrkraft in Form von Emoticons einen Gemütszustand (z.B. erschöpft, stolz, begeistert) und die Lerner*innen wenden sich spontan an eine ihnen räumlich nahe stehende Person und verbalisieren ihre Frage bzw. empfangen eine solche. An dieser Stelle wird unaufgefordert auch Mimik und Gestik eingesetzt, deren Betrachtung ebenfalls sehr interessant wäre.

- Ebenfalls als eine Art Sprechtheater zu kategorisierende, ist folgende, anspruchsvollere Übung. Ein wenige Zeilen umfassender Text -es kann sich auch um ein Gedicht handeln - wird für alle präsentiert. Auf Kärtchen werden Rollen verteilt, deren Stereotype eine sehr charakteristische Sprechweise innehaben (z.B. Soldat, Navigationssystem, Marktschreier). DieStudent ${ }^{\star}$ innen, die dieselbe Rollehaben, finden zusammen und besprechen, welche Eigenschaften der Sprechweise zugeordnet werden, mittels welcher prosodischen Gestaltung dies umzusetzen ist und üben ihre Vortragsweise. Im Anschluss lösen sich die Expert ${ }^{\star}$ innengruppen auf und es formieren sich neue Konstellationen, bei denen je eine Sprechrolle inszeniert wird. Die Gruppe hat die Aufgabe zu erraten, um welche Person es sich handelt. Die Auswahlmöglichkeiten können zur Erleichterung bereits für alle ersichtlich vorgegeben werden.

Die vorgestellten dramapädagogischen Übungen regen die Student ${ }^{\star}$ innen an, sich emotional in der Fremdsprache auszudrücken und machen ihnen zugleich bewusst, dass ihre lernersprachlichen Äußerungen (un-beabsichtigte) emotionale Wirkungen bei Hörer*innen verursachen können.

Zur Anfertigung der Vertonung ihrer zu zweit erfundenen Geschichten beschäftigen sich die Lernerinnen zunächst intensiv mit deren Verschriftlichung. Die Texte werden auf ihren emotionalen Gehalt hin untersucht und es wird unter Rückgriff auf zuvor erprobte sprecherische Variationen eine hörerorientierte Versprachlichung geplant. Impulsgebende Fragestellungen können dabei lauten:

In welcher Stimmlage ist die Geschichte zu sprechen? Kann innerhalb des Textes variiert werden?

Wie sollte die Melodieführung am Äußerungsende sein?

Wo sind Pausen zu setzen?

Welche Textstellen sind besonders spannend/ dramatisch und sollten mit einem Tonhöhenanstieg markiert werden? Wo bietet sich eine Erhöhung/Verlangsamung der Sprechgeschwindigkeit an?

Wo ist ein Anstieg der Lautstärke effektvoll?

Welche Informationen in den Sätzen sollen Satzakzente tragen?

Gibt es Passagen wörtlicher Rede? In welcher Stimmlage werden diese gesprochen? In welchem Gemütszustand befinden sich die Figuren und wie äußert sich dieser in ihrer Stimme?

Den Lerner*innen wurde ausreichend Zeit gegeben, um den Text auf diese Fragen hin zu untersuchen, zu bearbeiten und Markierungen vorzunehmen. Es bietet sich an, Symbole für die einzelnen prosodischen Merkmale zur Verfügung zu stellen bzw. zu vereinbaren, um die Markierungen übersichtlich zu halten. In den entstandenen studentischen Arbeiten konnte eine sehr diverse Verteilung der Sprechrollen beobachtet werden. Mal wurde die Geschichte abschnittsweise alternierend von den Student*innen eingesprochen, mal in Erzähler*in und Figurenstimme unterteilt. Auch dass nur ein Student die komplette Geschichte allein las, kam vor. Für die Aufnahme kann jede beliebige Sprachaufnahme-Funktion im Smartphone genutzt werden. Meist verfügen diese bereits über vorinstallierte Apps. ${ }^{1}$

\subsection{Mehrwert der Vertonung}

Die Aufnahme des Gelesenen als zu einem späteren Zeitpunkt wiederholt abspielbares Produkt bietet im Gegensatz zum flüchtigen Vorlesen im Kurs einige bemerkenswerte Vorzüge. Zum einen besteht die Möglichkeit, dass die Student*innen die Aufnahme beliebig oft wiederholen, bis sie mit dem Ergebnis zufrieden sind. Auch können Teile (1) Bei Iphone beispielsweise eignet sich die Funktion „Sprachmemos“. 
einzeln aufgenommen und später zusammengeschnitten werden. So muss bei einem Versprecher oder einer von den Sprecher*innen selbst als nicht adäquat gelesen erachteten Textstelle nicht die gesamte Geschichte erneut eingesprochen werden. Die Student ${ }^{\star}$ innen wiederholen bestimmte Stellen teilweise sehr oft und es tritt eine Art Automatisierungseffekt ein. Das Wissen um die Dauerhaftigkeit der Audio-Datei bewirkt zudem in den meisten Fällen eine gesteigerte Bemühtheit, was wiederum zu einem erhöhten Leistungsaufwand und besseren Ergebnissen in der prosodischen Gestaltung führen könnte. Zudem lassen sich zusätzlich mit Apps wie beispielsweise Spreaker ${ }^{2}$ spezielle Audio-Effekte einfügen. Das Hörbuch bekommt dann Hörspiel-Charakter. Ganz besonders zu unterstreichen ist noch der Aspekt der Abspielbarkeit der Vertonung, die es den Sprecher*innen ermöglicht, ihre eigene Sprechweise wahrzunehmen. Sich selbst in Aufnahmen zu hören, ist (auch in der Muttersprache) bekanntlich ungewohnt und teils sehr befremdlich. Häufig hat man den Eindruck, dass die gehörte Stimme nicht mit dem Klang der inneren Stimme beim Sprechen übereinstimmt. Es ist daher sehr förderlich, wenn Lernerinnen sich häufiger mit ihrer eigenen Stimme aus der Perspektive desder Zuhörerin konfrontiert sehen, sich an ihren Stimmklang in der Fremdsprache gewöhnen und ihr auf diese Weise eine positivere Einstellung gegenüber hegen.

\section{Schlussbemerkung}

Es sollte deutlich gemacht werden, dass das Thematisieren von Prosodie im Unterricht Deutsch als Fremdsprache wesentlich, da von hoher kommunikativer Relevanz ist. Lerner*innen sollten sich bereits im Anfängerunterricht und auch noch auf fortgeschrittenem Niveau mit der prosodischen Gestaltung von Äußerungen auseinandersetzen. Dafür sind zunächst Wahrnehmungsübungen und Hörtrainings vonnöten, um die muttersprachlichen Hörmuster zu erweitern. Lerner*innen sind zum selbständigen Einsatz von Prosodie spielerisch mittels dem Sprechtheater sehr nahestehenden rhetorischen Übungen hinzuführen. Besonderes Augenmerk ist dabei auf emotionales Sprechen zu richten, um den Lerner*innen den Zusammenhang zwischen prosodischer Gestaltung und Sprechwirkung vor Augen zu führen. Mit wachsenden Sprachkenntnissen sollte auch auf suprasegmentaler Ebene eine zunehmende Progression erfolgen, beispielsweise ist der Sprechakt Ironie und dessen stimmliche Signale sehr komplex und anspruchsvoll und lässt sich erst auf hohem Sprachniveau behandeln.

Die Vertonung von Geschichten stellt eine Unterrichtsaktivität dar, in der sich Lernerinnen auf kreative und intensive Weise mit unterschiedlichen Sprechweisen beschäftigen. Im Zuge der Vorbereitung der eigentlichen Aufnahmen sind prosodische Elemente und deren Wirkung immer wieder bewusstzumachen und selbst zu erproben. Natürlich lässt sich diese Aktivität der begrenzten Unterrichtsstunden geschuldet nicht allzu oft im Semester wiederholen. Das wäre sicherlich auch im Sinne eines abwechslungsreichen Unterrichts nicht empfehlenswert. Allerdings können im DaFUnterricht regelmäßig auch kürzere Texte bzw. Textabschnitte aus der aktuellen Lektion ausdrucksvoll vorgelesen und schließlich als Hausaufgabe aufgenommen und der Lehrkraft zugeschickt werden. Wünschenswert sind motivierende Aufgabenformate, bei denen ein bewusster Einsatz von Prosodie zur Vermeidung unbeabsichtigter Sprechwirkung im Vordergrund steht.

Literaturverzeichnis

\footnotetext{
Alvarez-Finkbeiner, M. (2003): „Lateinamerikanisches Spanisch“, in Hirschfeld, U.; Kelz, H.P.; Müller, U. (Hrsg.) (2013), Phonetik international: von Afrikaans bis Zulu. Kontrastive Studien für Deutsch als Fremdsprache. (online).

[http://www.phonetik-international.de/pphonetik].

Cohrs, S. (2007): „Wirkung und Akzeptanz prosodischer Interferenzen ungarischer Deutschlehrer auf deutsche Muttersprachler und ungarische Germanistikstudenten“, in Zeitschrift für Interkulturellen

Fremdsprachenunterricht (online). 12/2. [https://tujournals.ulb.tu-darmstadt.de/ index.php/zif/article/view/301/293].

Grzeszczakowska-Pawlikowska,

(2016): „Hörerorientierung in der rhetorischen "Redepraxis bei polnischen Germanistikstudierenden", in Hirschfeld, U.; Lange, F.; Stock, E. (Hg.), Phonetische und rhetorische Aspekte der interkulturellen Kommunikation. Schriften zur Sprechwissenschaft und Phonetik. Band 7. Frank \& Timme, Berlin, 77- 84.

Hirschfeld, U.; Reinke, K. (2018): ,Phonetik

im Fach Deutsch als Fremd- und Zweitsprache. Unter Berücksichtigung des Verhältnisses von Orthografie und Phonetik. 2. Aufl. Erich Schmidt Verlag, Berlin.

Hirschfeld, U. (2003): „Spanisch“, in Hirschfeld, U.; Kelz, H.P.; Müller, U. (Hrsg.) (2003ff), Phonetik international: von Afrikaans bis Zulu. Kontrastive Studien für Deutschals Fremdsprache. (online). [http://www.phonetik-international.de/pphonetik].

Hirschfeld, U. (1995): „Phonetische Merkmale 
in der Aussprache Deutschlernender und deren Relevanz für deutsche Hörer“, in Deutsch als Fremdsprache. Zeitschrift zur Theorie und Praxis des Deutschunterrichts für Ausländer. 32, 3/1995, 177 - 183.

Khider, A. (2019): Deutsch für alle. Carl Hanser, München

Neuber, B. (2006): „Phonetische und rhetorische Wirkungen sprechstimmlicher Parameter", in Deutsch als Fremdsprache. Zeitschrift zur Theorie und Praxis des Deutschunterrichts für Ausländer. 43, 3/2006, 151-156.

Reinke, K. (2011): ,Fremder Akzent - von der auditiven Wahrnehmung zur Deutung der Persönlichkeit“, $\quad$ in Babylonia. Die Ausspracheschulung im Fremdsprachenunterricht, 2/2011, 73-79.

Reinke, K. (2007): ,Zur emotionalen Wirkung phonetischer Mittel bei DaF-Lernenden“, in Zeitschrift für Interkulturellen Fremdsprachenunterricht: Didaktik und Methodik im Bereich Deutsch als Fremdsprache. (online) 12/2.

[https://tujournals.ulb.tu-darmstadt.de/ index.php/zif/article/view/378/366].

Sartor, E. (2007): „Phonetikund darstellendes Spiel: Zwei Stiefkinder begegnen sich im Unterricht Deutsch als Fremdsprache“, in Zeitschrift für Interkulturellen Fremdsprachenunterrich (online) 12:2.

[https://tujournals.ulb.tu-darmstadt.de/ index.php/zif/article/view/313/303].

Sperfeld, I. (2007): „Individualisierung im Phonetikunterricht. Die Arbeit mit Portfolios zur Unterstützung des Ausspracheerwerbs", in Zeitschrift für Interkulturellen Fremdsprachenunterricht (online) 12:2.

[https://tujournals.ulb.tu-darmstadt.de/ index.php/zif/article/view/303/295].

Travkina, E. (2007): „Zur Arbeit mit dem Hörbuch im Phonetik-Unterricht", in Zeitschrift für Interkulturellen Fremdsprachenunterricht (online) 12:1. [https://tujournals.ulb.tu-darmstadt.de/ index.php/zif/article/view/311/301].

Valman, G. (2016): „Unbetonte Silben als Lernproblem bei spanischsprachigen Deutschlernenden", in Hirschfeld, U. Lange, F.; Stock, E. (Hg.), Phonetische und rhetorische Aspekte der interkulturellen Kommunikation. Schriften

Sprechwissenschaft und Phonetik. Band 7. Frank \& Timme, Berlin, 267 - 275. 